\title{
Glomus tumor of small intestine: case report and review of literature
}

\author{
Yan Tan ${ }^{1}$, Xinyi Wang ${ }^{1}$, Guifang Yang ${ }^{1}$, Lan Liu ${ }^{1}$, Jun Fang ${ }^{1}$, Ying Chang ${ }^{1}$, Qiu Zhao , \\ and Yafei Zhang ${ }^{1}$ \\ ${ }^{1}$ Wuhan University
}

May 5, 2021

\begin{abstract}
Glomus tumors are exceedingly rare tumors arising from the normal glomus body. Only a very few cases located in the small intestine were reported to date. Here, we present a case of glomus tumor of the jejunum in a 30-year-old female patient, and discuss its clinical, imaging, and pathologic features.
\end{abstract}

\section{Glomus tumor of small intestine: case report and review of literature}

Yan Tan ${ }^{1,2}$, Xinyi Wang ${ }^{1,2}$, Guifang Yang $^{3}$, Lan Liu $^{1,2}$, Jun Fang ${ }^{1,2}$, Ying Chang ${ }^{1,2}$, Qiu Zhao ${ }^{1,2}$, Yafei Zhang ${ }^{1,2}$

${ }^{1}$ Department of Gastroenterology, Zhongnan Hospital of Wuhan University, Wuhan, China

${ }^{2}$ Clinical Center and Key Lab of Intestinal and Colorectal Diseases of Hubei Province, Wuhan, China

${ }^{3}$ Department of Pathology, Zhongnan Hospital of Wuhan University, Wuhan, China

\section{Correspondence}

Yafei Zhang, Department of Gastroenterology, Zhongnan Hospital of Wuhan University, No.169 Donghu Road, Wuhan 430071, China

Email: zhangyafei1127@163.com

\section{Key clinical message}

Glomus tumors rarely involve small intestine, with only 8 cases reported in the English literature up to date. In this paper, we presented the ninth case of small intestine glomus tumor in a 30-year-old female patient and analyzed associated clinical, radiographic, endoscopic and pathologic findings.

Abstract

Glomus tumors are exceedingly rare tumors arising from the normal glomus body. Only a very few cases located in the small intestine were reported to date. Here, we present a case of glomus tumor of the jejunum in a 30-year-old female patient, and discuss its clinical, imaging, and pathologic features.

Keywords: glomus tumor, small intestine, case report

\section{INTRODUCTION}

Glomus tumors (GTs) account for approximately $2 \%$ of all soft tissue neoplasms. ${ }^{1}$ However, rare visceral GTs do occur. GTs within the gastrointestinal tract have been reported to usually occur at the gastric, 
especially gastric antrum. The vast majority of gastric GTs follow a benign clinical course, without histologic or clinical evidence of malignancy. ${ }^{2}$ GTs rarely involve small intestine, with only 8 cases reported in the English literature, among which 4 cases present malignant features. ${ }^{3-5}$ Here, we report a patient with glomus tumor of the jejunum and review the literature on the clinicopathologic features, diagnosis and treatment.

\section{CASE PRESENTION}

A 30-year-old female referred to our hospital with the chief complain of melena and fatigue for 3 weeks. There were no significant positive signs other than anemic appearance. Routine blood examination showed that her hemoglobin level was $6.7 \mathrm{~g} / \mathrm{dL}$. Contrast-enhanced computed tomography (CT) revealed an obvious arterially enhancing mass in the small intestine and continuous enhancement in the delayed phase of enhancement (Figure 1). Capsule endoscopy indicated a possible mucosal eminence in the jejunum (Figure 2A). Because the photographs captured by capsule endoscopy were limited, oral single-balloon enteroscopy was further applied to determine the mass. After insertion of about $250 \mathrm{~cm}$ past pylorus, a $2.0^{*} 2.0 \mathrm{~cm}$ tumor without epithelial lining was discovered (Figure 2B). The patient was then transferred to the Department of Gastrointestinal Surgery and underwent a partial enterectomy.

At surgery, a $3.5 \mathrm{~cm}$ long segment of jejunum was removed. Gross pathology showed a $2.0 * 1.3 * 1.3 \mathrm{~cm}$ grayred polypoid protuberance in the small intestinal mucosal surface (Figure 3A). HE stain illustrated that the tumor was well limited by a thin fibrous capsule, interspersed with congestive capillaries of various size, and admixed with smooth muscle bundles (Figure 3B and 3C). Muscularis propria and serosa didn't show any damage. Immunohistochemical study showed that the tumor cells were strongly positive for SMA and collagen type IV (Figure 3D and 3E), and negative for CD31, CD34, CD117, Desmin, DOG-1, CgA, Syn, S100, EMA, STAT6, $\beta$-catenin, Caldesmon and Calponin. Ki-67 proliferation index was less than 1\% (Figure $3 \mathrm{~F})$ and mitotic activity is about $1 / 50 \mathrm{HPF}$.

According to the CT results, endoscopic images and pathologic findings, the case was finally diagnosed as benign GT. Postoperatively, the gastrointestinal hemorrhage was completely resolved. And no recurrence or metastasis was observed during a follow-up period of 6 months.

\section{DISCUSSION}

GTs arising from the small intestine are extremely rare. So far, only 8 cases have been described in the English literatures. ${ }^{3-5}$ Among them, two cases located at the duodenum, one case at jejunum, and five cases at the ileum. The clinicopathologic features of small intestinal GTs are summarized in Table 1. The case reported in our study is a 30-year-old female. Together with this case, the sex distribution is basically balanced (5 males and 4 females). Small intestinal GTs usually present non-specific symptoms such as abdominal pain and intestinal bleeding (melena or hematochezia). Larger tumors can cause intestinal stenosis and obstruction.

Due to the deep location and non-specific symptoms, small intestinal tumors including GTs are usually hard to differentiate and diagnose. Abdominal contrast-enhanced CT may be useful to distinguish GTs from other small intestinal tumors. According to previous description (mainly on gastric GTs), hemangioma-like globular enhancement with central fill-in and persistent homogeneous enhancement were both visualized in GTs. ${ }^{11}$ As for the reported case, the tumor on contrast-enhanced CT showed homogeneous high attenuation in the arterial, venous, and delayed phases. GTs in gastric usually display a submucosal pattern of growth. In the case reported by Knackstedt $\mathrm{C}$ et al., the GT located in duodenal bulb was polyp-like. ${ }^{7}$ Shelton JH et al. reported a GT of the ampulla, in which a protruding mass was seen. ${ }^{8}$ However, until now, there is no enteroscopic image presented in the jejunum or ileum GTs. In this paper, for the first time, we acquired the endoscopic imaging of small intestinal GT by balloon-assisted enteroscopy. Intriguingly, the enteroscopic imaging showed a fibrous capsule-covered mass, without any epithelial coverage, and on the surface of the tumor, no definite hemorrhage, erosions or ulcers were observed. Only some minor erosions on the area around the tumor edge were seen, which may cause the persistent melena.

Most GTs depend on postoperative pathological diagnosis. Under the microscope, the tumor consists of a 
large number of smooth muscle bundles and dilated capillaries. Immunohistochemically, tumor cells were stained positive for SMA and collagen type IV. Negative CD117 and DOG-1 expressions help in excluding the diagnosis of gastrointestinal stromal tumors, while negative CgA and Syn expressions help in excluding neuroendocrine neoplasms. ${ }^{12}$ Criteria for malignant GTs proposed by Folpe et al. include tumors with a deep location and a size of more than $2 \mathrm{~cm}$, or atypical mitotic figures, or moderate to high nuclear grade and [?] 5 mitotic figures $/ 50 \mathrm{HPF} .{ }^{13}$ According to the criteria, the present case was considered as low possibility of malignancy. And 6 months follow-up showed no indication of recurrence or metastasis after a partial enterectomy, which supporting the benign diagnosis.

All the small intestinal GTs recorded in the literature have received surgical resection, but no patients received regional lymphadenectomy. After surgery, also no patients received adjuvant radiotherapy or chemotherapy. However, 2 patients developed metastases in the 8 reported cases. Therefore, standardized systemic treatment needs to be further studied. For gastric GTs, endoscopic or laparoscopic resection is recommended. Also due to the fact of extremely rare cases of malignant GTs in gastric, no standardized neoadjuvant or adjuvant treatment was suggested. Radiotherapy or chemotherapy has been utilized for the treatment of malignant GTs of head and neck. However, in the reported cases, tumor progression was not reliably altered..$^{14}$

In conclusion, we have reported a case of GT of small intestine in a 30-year-old female patient. The final diagnosis is made by histological and immunohistochemical examination. Due to the exceedingly rare occurrence, there is no standardized management pathway for these patients. As there is a potential for malignancy, early diagnosis and treatment are important for a good prognosis.

\section{ACKNOWLEDGMENT}

The authors would like to thank the patient for agreeing to have her information published.

\section{CONFLICT OF INTEREST}

None declared.

\section{AUTHORS' CONTRIBUTIONS}

All authors participated in the case report.

\section{ETHICAL APPROVAL}

This study has been approved by the Ethics Committee of Zhongnan Hospital of Wuhan University and was conducted in accordance with the Helsinki Declaration.

\section{CONSENT FOR PUBLICATION}

Written informed consent was obtained from the patient for the publication of this case report and any accompanying images. A copy of the consent form is available for review by the Editor of this journal.

\section{DATA AVAILABILITY STATEMENT}

The data that support the findings of this study are available on request from the corresponding author. The data are not publicly available due to privacy or ethical restrictions.

\section{ORCID}

Yafei Zhang, https://orcid.org/0000-0003-3329-8029

\section{REFERENCES}

1. Gombos Z, Zhang PJ. Glomus tumor. Arch Pathol Lab Med . 2008;132(9):1448-1452.

2. Mago S, Pasumarthi A, Miller DR, Saade R, Tadros M. The two challenges in management of gastric glomus tumors. Cureus . 2020;12(7):e9251. 
3. Chen JH, Lin L, Liu KL, et al. Malignant glomus tumor of the intestinal ileum with multiorgan metastases: A case report and review of literature. World J Gastroenterol . 2020;26(7):770-776.

4. Ma YH, Li P, Jiang GZ, Jin RJ, Li WC. Gastrointestinal glomus tumors: a clinicopathological analysis of fifteen cases. Zhonghua Bing Li Xue Za Zhi . 2020;49(1):22-27. (Article in Chinese)

5. Campana JP, Goransky J, Mullen EG, Palavecino EM. Intestinal benign glomus tumor: description and review of the literature. Dig Dis Sci . 2014;59(10):2594-2596.

6. Abu-Zaid A, Azzam A, Amin T, Mohammed S. Malignant glomus tumor (glomangiosarcoma) of intestinal ileum: a rare case report. Case Rep Pathol . 2013;2013:305321.

7. Knackstedt C, Wasmuth H, Donner A, Trautwein C, Winograd R. Diagnosis of an unusual tumor in the duodenum. Endoscopy . 2007;39(Suppl 1):E94.

8. Shelton JH, Lamont JP, Zieske A, Mallat DB. Glomus tumor of the ampulla. Gastrointest Endosc . 2007;66(2):395-396; discussion 396.

9. Geraghty JM, Everitt NJ, Blundell JW. Glomus tumour of the small bowel. Histopathology . 1991;19(3):287-289.

10. Hamilton CW, Shelburne JD, Bossen EH, Lowe JE. A glomus tumor of the jejunum masquerading as a carcinoid tumor. Hum Pathol . 1982;13(9):859-861.

11. Hur BY, Kim SH, Choi JY, et al. Gastroduodenal glomus tumors: differentiation from other subepithelial lesions based on dynamic contrast-enhanced CT findings. AJR Am J Roentgenol . 2011;197(6):1351-1359.

12. Grande C, Haller DG. Gastrointestinal stromal tumors and neuroendocrine tumors. Semin Oncol Nurs . 2009;25(1):48-60.

13. Folpe AL, Fanburg-Smith JC, Miettinen M, Weiss SW. Atypical and malignant glomus tumors: analysis of 52 cases, with a proposal for the reclassification of glomus tumors. Am J Surg Pathol . 2001;25(1):112.

14. Wolter NE, Adil E, Irace AL, et al. Malignant glomus tumors of the head and neck in children and adults: Evaluation and management.Laryngoscope . 2017;127(12):2873-2882.

\section{FIGURE LEGENDS}

FIGURE 1 Features of the GT on contrast-enhanced CT. (A, B) CT images during arterial phase (coronal and horizontal planes) showed an obvious enhanced nodule in the small intestine (arrow head); (C, D) CT images during delayed phase (coronal and horizontal planes)

FIGURE 2 Endoscopic features of the GT. (A) Image of capsule endoscopy; (B) Image of oral single-balloon enteroscopy

FIGURE 3 Gross findings, histological and immunohistochemical characteristics of the GT. (A) Gross image of resected mass; (B) H\&E, $\times 40$; (C) H\&E, $\times 100$; (D) SMA immunostaining, $\times 200$; (E) Collagen type IV immunostaining, $\times 200$; (F) Ki-67 immunostaining, $\times 400$ 

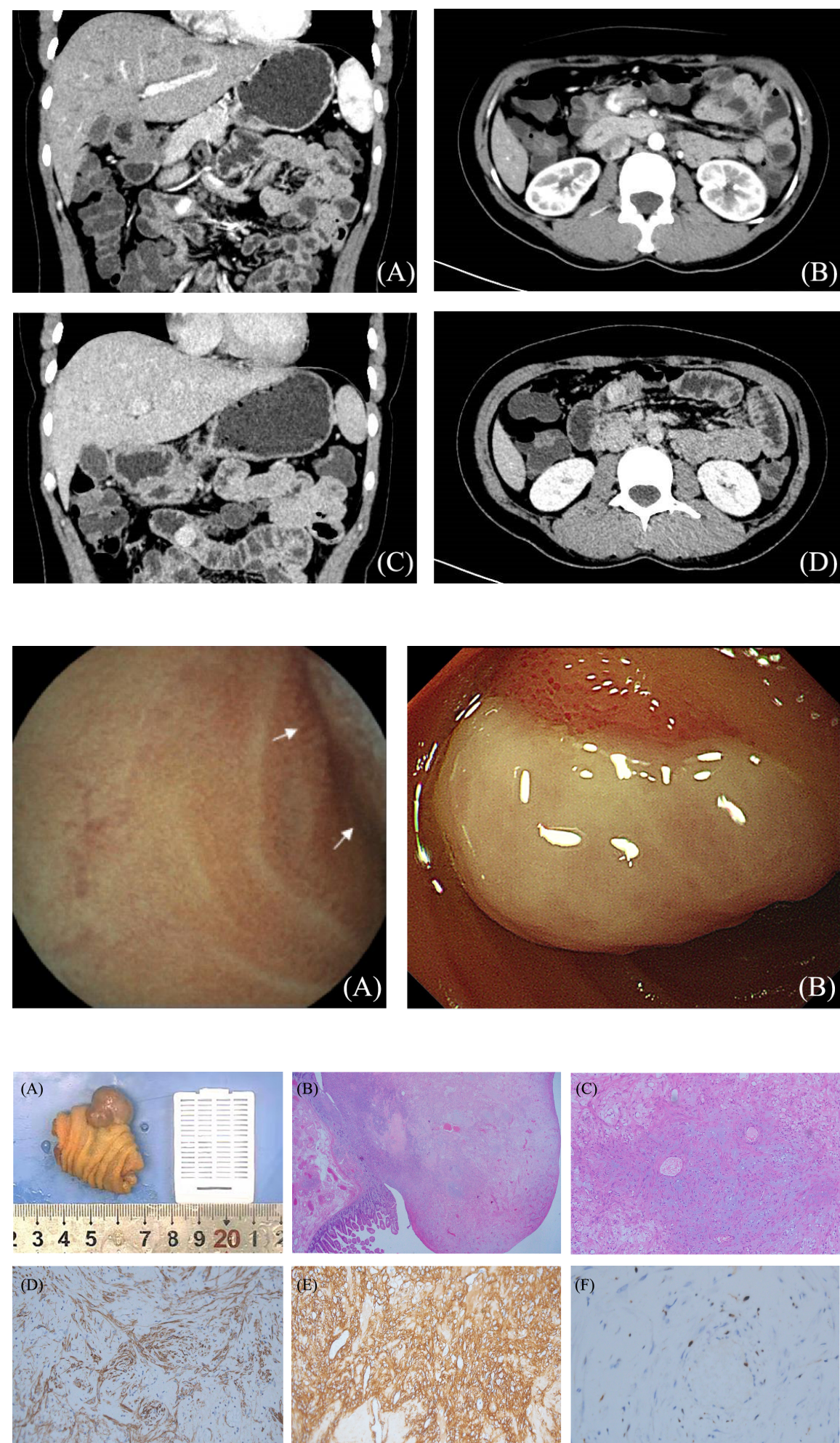